\title{
Is there an alignment of quasars near NGC 520 ?
}

\author{
E. Gosset, J. Surdej, and J.P. Swings \\ Institut d'Astrophysique, University of Liège, Belgium
}

\section{Abstract :}

Three statistical tests applied to 65 UV excess objects in the field of NGC 520 lead to no detection of any significant alignment.

\section{Introduction}

In 1985 we (Gosset et al, 1986) reported on the results of several statistical analyses of a 25 square degree field around NGC 450, i.e. essentially on the detection of a clustering on a scale of order 10 arc minutes. The present contribution deals no longer with clusterings per se, but with searches for possible alignment(s) in another field containing an irregular galaxy, NGC 520.

The field of NGC 520 was chosen following the claim by Arp and Duhalde (1985) that "six of the seven nearest quasars to NGC 520 are aligned so accurately across the galaxy as to demonstrate physical association".

The three statistical tests briefly described below were applied to a sample of 65 ultraviolet excess objects (including 48 bona fide quasars) detected in a $4.5^{\circ} \times 4.5^{\circ}$ field around NGC 520, on the basis of U/B dual image Schmidt plates (see Swings et al, 1985; Gosset et al, 1986). As explained on several occasions (see e.g. Gosset et al, 1986), the "plates have been scanned at least four times by two different persons in an unbiased homogeneous and objective manner". 


\section{Statistical tests}

The three analyses performed on the 65 objects and the respective results are the following :

i) the blunt angle technique, described by Kendall and Kendall (1980) and Webster (1982). The objects are taken 3 by 3 to form triangles : for each of these, the minimum angle (bluntness) among the supplements of the angles at the vertices is selected; the statistic is a function of the distribution of those minimum angles. Even if the tolerance on the alignment angle is relaxed to 1 degree, no alignment is seen in the field of NGC 520 to better than 1 standard deviation;

ii) the spherical blackboard technique, developed by Kendall (1984). The shape of a triangle belonging to a plane can be represented by one point in a shape-space whose property is to be isometric with a two-dimensional sphere. The distribution of shapes can therefore be investigated through the distribution of the representative points on the above-mentioned sphere. In particular, the locus of the triangles that degenerate into a line is well defined. For the 43680 points ( 65 objects taken 3 by 3 ) of our sample, only a minor, almost insignificant, deviation from randomness occurs but its location on the spherical blackboard ( $L$ vertex of figure 2 of Kendall (1984)) corresponds to a degeneracy by coincidence of two vertices of the triangles. This clearly indicates a tendency for the objects to cluster and not to line up. Consequently, no statistically significant alignment is found by a three points'statistic in our sample of quasars or quasar-candidates in the field of NGC 520;

iii) a third method derived from a pattern recognition method developed for the automated detection of particle tracks in bubble chambers, has been adapted by one of $u s(E G)$. to serve as a statistical test for alignments. The basic idea is simple; each "point" (the object) is transformed into a "line" such that several aligned "points" will give rise to "lines" going through the same intersection point. In the space of the intersection points, an approximate alignment will correspond to a cluster : such a space can be investigated using a hierarchical clustering method like, for example, the single linkage cluster analysis based on the minimum spanning tree (MST, Gower and Ross, 1969). A statistic based on the MST edge-length distribution is actually very powerful, but this test leads to the detection of no significant alignment in the present case. 


\section{Conclusion}

On the basis of the three tests described above, it is concluded that, as far as our sample is concerned, no significant alignment is detected in a field of $4.5^{\circ} \times 4.5^{\circ}$ containing NGC 520.

\section{References}

ARP, H., DUhaldE, 0. : 1985, Publ. Astron. Soc. Pac., 97, 1149.

KENDALL, D.G., KENDALL, W.S. : 1980, Adv. Appl. Prob., 12, 380.

KENDALL, D.G. : 1984, Bull. London Math. Soc., 16, 81.

GOSSET, E., SURDEJ, J., SWINGS, J.P. : 1986, in Quasars, IAU Symp. 119, eds. : G. SWARUP, V.K. KAPAHI, 45.

GOWER, J.C., ROSS, G.J.S. : 1969, Appl. Statistics, 18, 54.

SWINGS, J.P., SURDEJ, J., HENRY, A., GOSSET, E., ARP, H. : 1985, Rev. Mexicana Astron. Astrofis., 10, 91.

WEBSTER, A. : 1982, Monthly Not. Roy. Astron. Soc., 201, 179.

\section{DISCUSSION}

WAMPLER: If you created a perfect line of 5-10 quasars and added it to your observed field would your 3-point statistical tests find or notice it?

SWINGS: The first method is not sensitive to the perfection of the line and, in our particular case, will require approximately eight to nine quasars to reach the $95 \%$ confidence limit. The second one will detect the perfection of the line (presence of the shape-points on the LM edge of the spherical blackboard); however, concerning the number of aligned objects, the computation of the probability will suffer from the same difficulties as the first method. An increase of the sample may add overwhelming noise to the possible signal.

The third method is not a three-point statistical test; it is sensitive to the number of aligned objects but also reacts continuously to the perfection of the line. Six truly aligned quasars would be detected to approximately the 3 standard deviations' level.

ARP: This elaborate statistical test has been performed on a set of 
quasars and candidates which are not relevant to the association with NGC 520. NGC 520 is the second brightest very disturbed galaxy in the sky. The quasars which are associated with it are conspicuously bright. If you refer to the picture I show here (from P.A.S.P. 1986) you see that just the confirmed quasars brighter than 19.0 magnitude form the line through NGC 520.

Five of the quasars on this line were found from the original searches of Swings and Surdej. Subsequent searches of the region on plates taken with a different telescope by Arp and Duhalde revealed one more quasar on the line and 5 off the line. This is one of the most completely searched regions anywhere in the sky that I know of. One can judge for oneself by looking at the picture whether or not the existence of the line needs to be tested.

SWINGS: I can only restate the conclusion of the work described here, $i$.e. that as far as our homogeneous sample is concerned, no significant alignment is detected in a $4.5^{\circ} \times 4.5^{\circ}$ field containing NGC 520 . 